\title{
LES DIRECTEURS·TRICES DE CENTRES SOCIAUX FACE À LA BUREAUCRATISATION
}

Les conditions de travail dans le monde associatif dépendantes de l'action des pouvoirs publics?

\section{Simon Cottin-Marx, Emmanuelle Paradis}

Association RECMA | « RECMA »

2020/3 N³57 | pages 109 à 120

ISSN 1626-1682

DOI 10.3917/recma.357.0109

Article disponible en ligne à l'adresse :

https://www.cairn.info/revue-recma-2020-3-page-109.htm

Distribution électronique Cairn.info pour Association RECMA.

(C) Association RECMA. Tous droits réservés pour tous pays.

La reproduction ou représentation de cet article, notamment par photocopie, n'est autorisée que dans les limites des conditions générales d'utilisation du site ou, le cas échéant, des conditions générales de la licence souscrite par votre établissement. Toute autre reproduction ou représentation, en tout ou partie, sous quelque forme et de quelque manière que ce soit, est interdite sauf accord préalable et écrit de l'éditeur, en dehors des cas prévus par la législation en vigueur en France. Il est précisé que son stockage dans une base de données est également interdit. 


\title{
LES DIRECTEURS·TRICES DE CENTRES
} SOCIAUX FACE À LA BUREAUCRATISATION

LES CONDITIONS DE TRAVAIL DANS LE MONDE ASSOCIATIF DÉPENDANTES DE L'ACTION DES POUVOIRS PUBLICS?

\author{
par Simon Cottin-Marx* et Emmanuelle Paradis**
}

I es pouvoirs publics ont toujours imprimé leur marque sur le monde associatif employeur, qu'ils financent et réglementent largement (Hély, 2009 ; Cottin-Marx, 2019). Grâce aux travaux de Viviane Tchernonog et de Lionel Prouteau (2019), nous savons que les rapports entre la puissance publique et les associations se sont considérablement transformés ces dernières années, en particulier depuis la crise économique de 2008, et qu'ils ont été marqués par la stagnation des financements publics et le développement de la logique contractuelle (Lipsky et Smith, 1989-1990). Ces évolutions n'ont pas été neutres pour les associations. Pour ces auteurs, elles ont entraîné le déplacement des projets associatifs vers des publics plus solvables, réduit la capacité des organisations loi 1901 à expérimenter et à innover, et conduit à la concentration des financements publics dans les grandes associations, "qui ont la taille critique et les ressources humaines suffisantes pour accéder à ces formes de financements » (Tchernonog et Prouteau, 2017). Une situation qui exclut de fait les associations employeuses de taille plus limitée des circuits du financement public et a entraîné la disparition d'un grand nombre d'entre elles : entre 2011 et 2017, le nombre d'associations employeuses est passé de 182000 à 159000 (-13\%).

$\mathrm{Si}$, grâce aux auteurs du Paysage associatiffrançais, les conséquences de ces évolutions sur les associations sont documentées, les effets sur les salariés et l'organisation du travail sont moins étudiés par les chercheurs. Quels ont été les résultats de cette dynamique, alimentée par la crise économique de 2008, sur le travail dans les associations? Au moment de la rédaction de cet article, en mai 2020, cette question prend une acuité nouvelle. Car tout semble indiquer qu'une nouvelle crise économique (et des finances publiques) va se surajouter à la crise sanitaire que nous traversons en raison de la pandémie de Covid-19. Il est aujourd'hui difficile d'en prédire les contrecoups. Cependant, en interrogeant la crise passée et ses conséquences nous espérons en tirer des enseignements pour celle qui commence.

* Sociologue, chercheur associé au Latts

** Cheffe de projet « Prévention et santé au travail », Chorum-Cides 
Pour répondre à notre question, nous nous appuierons sur l'analyse d'une enquête réalisée auprès de directeurs-trices de centres sociaux : que nous disent-ils de l'évolution de leur métier et de leurs conditions de travail ? Après avoir présenté notre objet et notre méthode (I), nous montrerons que les directeurs.trices voient leur travail se transformer, qu'ils sont confrontés à un "saupoudrage » des financements publics qui représente pour eux un véritable fardeau administratif (II). Puis nous analyserons les conséquences de cette bureaucratisation sur la santé au travail et l'efficacité des associations (III).

\section{Présentation de l'enquête auprès des directeurs-trices de centres sociaux}

Pour éclairer les effets sur le travail associatif de la transformation $\mathrm{du}$ " partenariat » entre pouvoirs publics et associations, nous exploiterons les données d'une enquête menée en $2018^{1}$, par la mutuelle Chorum ${ }^{2}$ auprès de 165 directeurs.trices de structures de la branche des acteurs du lien social et familial. Ces responsables d'associations employeuses, comprenant entre 3 et 60 salariés, ont été interrogés sur les sources de satisfaction et d'insatisfaction dans leur travail et sur les évolutions de leurs conditions de travail ces dernières années.

Le point de départ de cette enquête est le constat d'une détérioration de la santé des directeurs.trices des associations relevant de cette branche (centres sociaux, crèches, associations de développement social local). Ce constat, effectué par Chorum à partir des données recueillies en tant que mutuelle de prévoyance et conforté par les éléments rassemblés par la branche, a été mis en lien avec les résultats d'une enquête de la branche sur l'exposition des salariés aux risques psychosociaux (RPS). Dans ce contexte, le Snaecso ${ }^{3}$ a décidé de confier à Chorum la réalisation d'une enquête sur les conditions de travail des directeurs-trices des structures de la branche, à partir de leurs réponses à des questions ouvertes sur leurs satisfactions et insatisfactions au travail, et sur leur vision de l'évolution passée et à venir de leur activité.

\section{Le métier de directeur-trice de centre social}

Si l'on se réfère à la convention collective nationale des acteurs du lien social et familial ${ }^{4}$, dont relèvent les centres sociaux, les directeurs-trices ont pour charge d'assumer la responsabilité générale de la structure par délégation du conseil d'administration. Ils occupent une place stratégique qui les amène à participer "activement au projet de l'association", dont ils sont garants de la mise en œuvre. Ils sont "responsables ou coresponsables de l'administration générale, de la gestion de la structure et des ressources humaines ainsi que de la recherche de financement » et donc occupent la «fonction employeur " : ils sont amenés à diriger, à assumer et à coordonner la ou les structures, les équipes ainsi que l'animation globale. Ils assurent
(1) « Les conditions de travail des directeurs.trices de centres sociaux, EAJE et ADSL », 24 août 2018.

(2) Chorum est l'offre de protection sociale complémentaire destinée à l'économie sociale et solidaire. Depuis 2007, elle accompagne les branches de l'ESS et ses adhérents dans leurs démarches de santé et de qualité de vie au travail ainsi que dans la prévention des risques professionnels.

(3) Syndicat employeur de la branche des acteurs du lien social et familial, aujourd'hui Elisfa.

(4) «Convention collective nationale des acteurs du lien social et familial : centres sociaux et socioculturels, associations d'accueil de jeunes enfants, associations de développement social local », 4 juin 1983. 
aussi " une fonction de veille et de conseil aux élus ", "participent au développement local et à la promotion de la vie associative». Et enfin, ce qui n'est pas la moindre de leurs missions, ils « recherchent et développent des partenariats extérieurs et travaillent en réseau ». Cette " fiche de poste » indique les grandes lignes des tâches que réalisent les directeurs·trices. Évidemment, la réalité du travail varie d'une structure à l'autre, en fonction d'une multitude de variables (missions réalisées par la structure, gouvernance, structure salariale, etc.).

Il faut également envisager ces missions dans le contexte, décrit plus haut, de très petites structures. Les directeurs·trices sont souvent également animateurs de l'activité; ils pilotent des équipes en partie peu professionnalisées (contrats aidés), sans soutien technique interne à la structure (fonctions supports).

\section{Les conditions de travail des directeurs $\cdot$ trices de centres sociaux}

L'enquête réalisée auprès des directeurs et des directrices de centres sociaux nous permet de repérer leurs principaux motifs de satisfaction et d'insatisfaction au travail.

\section{Satisfaction et insatisfaction au travail des directeurs •trices de centres sociaux}

\begin{tabular}{|c|c|c|c|}
\hline Thèmes de satisfaction & Thèmes d'insatisfaction & \\
\hline Sens du travail & $32,6 \%$ & Charge de travail & $54,8 \%$ \\
\hline Contenu du travail & $22,9 \%$ & $\begin{array}{c}\text { Injonctions } \\
\text { institutionnelles }\end{array}$ & $11,8 \%$ \\
\hline Dynamique collective & $18,2 \%$ & Baisse des financements & $6,3 \%$ \\
\hline Animation d'équipe & $13,8 \%$ & Animation d'équipe & $5,4 \%$ \\
\hline $\begin{array}{c}\text { Reconnaissance } \\
\text { institutionnelle }\end{array}$ & $4,8 \%$ & $\begin{array}{c}\text { Solitude, manque de } \\
\text { soutien }\end{array}$ & $3,9 \%$ \\
\hline Gestion financière & $3,7 \%$ & $\begin{array}{c}\text { Recherche de } \\
\text { financements }\end{array}$ & $3,2 \%$ \\
\hline Relations CA/bureau & $3,3 \%$ & Gestion RH & $2,4 \%$ \\
\hline Évolutions positives & nonévoqué & $\begin{array}{c}\text { Baisse du sentiment } \\
\text { d'utilité }\end{array}$ & $0,9 \%$ \\
\hline Rémunération & 0,6 $\%$ & Rémunération & $0,3 \%$ \\
\hline Conditions de travail & Nonévoqué & Conditions de travail & $\begin{array}{c}\text { Gestion des } \\
\text { remplacements }\end{array}$ \\
\hline
\end{tabular}

Les principaux motifs de satisfaction au travail sont "le sens $d u$ travail, l'utilité pour les adhérents et usagers, les valeurs défendues, la finalitésociale, l'atteinte d'objectifs qualitatifs», "le contenu du travail, la diversitédes missions, la conduite de projets», "la dynamique collective avec les salariés et/ou les bénévoles, la convivialité, le travail collectif», «le pilotage du travail d'équipe, l'animation de l'équipe de salariés, leur motivation, l'appui à leurévolution personnelle».

Les principaux motifs d'insatisfaction sont, quant à eux, «la charge 
quantitative de travail, l'inflation des tâches vécues comme "administratives" et des e-mails, la pression", puis "les injonctions institutionnelles, les évolutions vécues comme négatives et la lourdeur administrative des tutelles et des financeurs", et enfin "la baisse des financements, l'absence de visibilité budgétaire et le manque de moyens matériels et humains ".

Dans la suite de cet article, nous allons nous intéresser principalement aux motifs d'insatisfaction. Où prennent-ils leur source? Que nous disent ces résultats de la transformation du métier de directeur.trice et des contraintes auxquels ils sont confrontés ? L'étude a permis de repérer des dynamiques similaires, transversales aux différentes structures.

(5) «Panorama2018delabrancheprofessionnelle des acteurs du lien social et familial », Observatoire emploi formation delabranche professionnelle Alisfa.

\section{Les associations étudiées dans l'enquête de Chorum}

L'enquête de Chorum portait sur les associations de la branche des acteurs du lien social et familial. La branche Alisfa rassemble principalement les centres sociaux (qui nous intéressent dans cet article), les établissements d'accueil de jeunes enfants (EAJE, notamment les crèches) et les associations de développement social local.

Le panorama $2018^{5}$ de cette branche, portant sur les données 2016, fournit les éléments suivants :

- 3690 structures, ayant très majoritairement le statut d'associations ;

- 89400 salariés, représentant 36400 équivalents temps plein (ETP);

- $71 \%$ des structures ont moins de 10 ETP, et $32 \%$ moins de 5 ;

- $13 \%$ des structures gèrent plusieurs établissements ;

\section{Qu'est-ce qu'un centre social?}

Selon l'Observatoire national des centres sociaux, "un centre social est un équipement de proximité (quartier, petite ville, canton...) ouvert à tous, tous âges et conditionséconomiques et sociales confondus ». Principalement implantés dans les territoires urbains, et en particulier dans les quartiers "politique de la ville», ils constituent un "dispositif d'action, d'animation et d'intervention dans la vie locale. Plus qu'un simple établissement, c'est un équipement polyvalent pour les habitants, leur offrant la possibilité de pratiquer un sport, de s'initier à l'informatique, de cuisiner, de consulter les services sociaux, etc. (Durand, 2006).

Les centres sociaux et les EAJE sont des structures à faible effectif salarié, ayant à leur tête un directeur.trice ou un.e responsable salarié. Une partie d'entre elles se rassemblent à un niveau municipal ou de communauté de communes, mais en faible proportion, car toutes ces associations ont pour principe une implication des usagers dans les instances, et donc une proximité forte avec le niveau local ou de quartier (Cortesero, 2013).

Dans la typologie des «entreprises associatives » proposée par Matthieu Hély (2009), les centres sociaux associatifs correspondent à l'idéal-type des "entreprises associatives partenaires ", car elles sont marquées par la forte présence de financements "non-marchands » et d'un salariat majoritairement «atypique». Sur ce dernier aspect, le sociologue Francis Lebon (2016) pointe la forte présence d'" emplois précaires » dans ces structures : "À l'échelle de l'ensemble des centres sociaux associatifs, environ la moitiédes salariés ne sont pas en CDI. » Une précarité qui concerne tous les salariés, y compris «les directeurs et, plus encore, les directrices». Une analyse que confirme l'observatoire de la branche Alisfa, qui constate que $52 \%$ seulement des salariés sont en CDI, et $40 \%$ à temps plein. 
(6) Un Cerfa est un formulaire administratif réglementé. Il s'agit d’un imprimé officiel dont le modèle est fixépararrêté. L’appellation provient du nom del'organisme public chargé d'éditer ces formulaires : le centre d'enregistrement et de révision des formulaires administratifs (Cerfa).
« Saupoudrage » des financements publics et " fardeau administratif » : les dynamiques à l'œuvre

Pour financer les emplois et les activités de leurs structures, les directeurs-trices de centres sociaux ont la responsabilité de trouver des financements, majoritairement publics comme nous l'avons vu. Pour cela, ils sont amenés à s'adresser aux différentes strates institutionnelles (Union européenne, État, Région, département, communauté d'agglomérations, ville, etc.), parfois sur plusieurs territoires (plusieurs communes par exemple) et à plusieurs administrations sectorielles d'une même collectivité, qui travaillent parfois de manière indépendante (" en silos»). À titre d'exemple, une structure pourra s'adresser au service " emploi et formation » de la Région pour des aides à l'emploi, au service « culture » pour soutenir une activité, et enfin au service « cohésion territoriale " pour une autre. Les dirigeants associatifs font face à un environnement institutionnel public complexe. De leur point de vue, cette multiplicité de guichets peut apparaître comme autant d'opportunités de financements. Cependant, les entretiens montrent qu'ils la voient surtout comme un "saupoudrage » de l'argent public (Bezes et Siné, 2011), suscitant un véritable «fardeau administratif» (Hall et al., 2005 ; Cottin-Marx, 2016). C'est le cas, par exemple, de ce directeur qui nous explique avoir été victime, en 2017, d'une véritable «surcharge administrative ». Au cours de l'année, sa structure avait bénéficié de pas moins de 27 sources de financements, "autant de dispositifs à concevoir, à accompagner et à évaluer».

La situation de ce directeur n'est pas isolée. Un autre regrette ainsi "la multiplicité des canaux et modes de réponse aux appels à projets (papier, numérique, nombre d'exemplaires, nombre de destinataires/de services...)». S’il constate que des évolutions récentes, comme la mise en place du Cerfa ${ }^{6}$ unique de demande de subvention, représentent « une amélioration pour les petites associations ne répondant qu'à des appels à projets locaux ou intercommunaux", il déplore que les structures "plus importantes ou ayant besoin de plus de fonds (qui répondent à des appels à projets émanant des départements, des Régions, de l'État, de l'Europe, de la CAF, de la MSA, de la Carsat) doivent remplirdes dossiers tous différents. En version numérique, parfois, mais mal construits; en ligne, d'autres fois, mais jamais sur la même plateforme et donc jamais dela même manière, pour répéter tous les ans la mêmechose sur la partie administrative (pas de mémoire des pièces annexes); en version papier, enfin. Et quand c'est en version numérique, il faut encore imprimer pour signer le document puis le scanner pour l'envoyer par mail ».

L'inadaptation des outils utilisés par les financeurs, si elle n'est pas systématique, semble récurrente. Un directeur évoque par exemple «les exigences de la CAF en termes de documents (12 pages de classeur Excel pour un compte de résultat), des tableaux à cellules verrouillées qui ne correspondentjamais à notre réalité». Un autre témoigne de la difficulté 
de "composer avec des directives publiqueset/ou politiques dontle raisonnement est uniquement financier » et dont "les programmes d'action et de financement [ne] s'inscrivent que [dans] des logiques annuelles ».

\section{La tendance à la complexification administrative vécue comme du " harcèlement "}

"L'absence de lisibilité» des financements, les « injonctions » institutionnelles et "des choix politiques qui bougent sans cesse» sont vécus par les directeurs comme ce que l'un d'eux qualifie de " harcèlement administratif». Ils sont nombreux à évoquer des exigences vécues comme inutiles et incohérentes. C'est le cas de ce responsable associatif qui explose littéralement à propos des « délais de rendu exigés par la CAF, qui, elle, traiteles dossiers deuxà trois mois plus tard» et du "décalage entre les niveaux d'attente CAF surnos démarches de projets sociaux et les écrits pilotés par la CAF elle-même, qui se résument à deux malheureuses questions. "Faites ce qu'on dit, pascequ'on fait!"”. Pour obtenir les ressources publiques nécessaires à l'équilibre de leur modèle économique, les associations doivent porter un lourd fardeau administratif résultant du morcellement de la puissance publique (Thoenig, 2005), mais aussi du fait que les différentes administrations susceptibles de les financer n'ont ni les mêmes attentes ni les mêmes calendriers et ne fonctionnent pas selon les mêmes modalités.

\section{Les financements publics : entre baisse et stagnation, retard et incertitude}

Comme nous l'avons vu plus haut, depuis la crise de 2008, les ressources publiques à destination des associations ont stagné (Tchernonog et Prouteau, 2019). Qui plus est, le mode d'attribution de ces financements s'est transformé: ceux-ci prennent de moins en moins la forme de subventions et davantage celle de marchés publics. L'enquête montre que ces changements sont ressentis par les directeurs-trices des centres sociaux comme pesant sur leurs tâches administratives. Certains déplorent ainsi : «La baisse des moyens est massive et proportionnellement inverse à l'augmentation des besoins. » Les entretiens révèlent aussi la montée d'un climat "d'incertitude». L'un témoigne avoir "de plus en plus de pression financière. Les institutions paient tardivement (gestion de la trésorerie) ». Un autre directeur nous confie : "Les financements en baisse ou tardifs (voire différés) génèrent une angoisse permanente : comment vais-je pouvoir payer les salariés, quelles actions doivent être abandonnées? Le moment des bilans annuels financiers est toujours perturbant, sachant que l'on n'a pas toujours une vue continue des engagements. Si l'on attend lesfinancements, on ne fait plus grand-chose. » Deux autres ajoutent: "Inquiétude ++ quant à la baisse régulière des moyens alloués (postes aidés), qui entrave toute visibilité à plus d'un an et génère dans les équipes un sentiment d'insécurité légitime. " "Une incertitude dans les projections financières, ce quifragilise la motivation des équipes. » Finalement, ce n'est pas tant la baisse ou la stagnation des ressources 
publiques qui inquiète les directeurs-trices, mais plutôt l'absence de perspectives claires, créant une incertitude anxiogène. Comme nous l'avons vu dans un précédent travail (Cottin-Marx, 2016 ), les fonctionnaires en charge d'attribuer les ressources publiques aux associations, sont eux aussi dans l'incertitude vis-à-vis de leurs propres budgets, ce qui a des conséquences par ricochet sur les associations. "Paradoxalement, les pouvoirs publics demandent aux associations d'être professionnelles, defaire des projections de leurs budgets sur trois ans, mais, chaque année, c'est l'incertitude sur leurs propres budgets. Ce qui maintient les associations dans l'instabilité et l'incertitude. »

\section{Contraction budgétaire et exigences publiques renforcées}

Outre la surcharge administrative qui découle du millefeuille administratif et de l'absence de coordination entre ces différentes couches d'acteurs publics, les directeurs-trices constatent une situation paradoxale : alors que les financements se contractent, les attendus pour obtenir des fonds publics deviennent plus importants. Comme l'explique un directeur, "à mesure que les financements se raréfient, les demandes de reporting se développent : la nouveauté est que les financeurs créent leurs outils de reporting là où, auparavant, un bilan qualitatifet quantitatif suffisait ». Et cette fois encore, chaque strate publique a des instruments qui lui sont propres: «Le principal problème, c'est que ces outils sont tous différents et que les indicateurs nesont pas les mêmes. Par exemple, on doit fournir le nombre de 26-50 ans à l'un et le nombre de 45-55 ans à un autre, etc. Nos activités concernent plus de 4000 personnes, il est difficile de retraiter les données en fonction des besoins des financeurs. Nous gérons 40 lignes definancements. Nous avons nos propres outils internes, mais nous n'avons pas fini la numérisation de nos activités et suivis. Et, de toutefaçon, il faudra quand même extraire les données pour remplir le tableau de tel financeur.»

Pour expliquer ce paradoxe, nous pouvons mobiliser les travaux de Béatrice Hibou $(2012$; 2013) ou encore de David Graeber (2015), qui analysent l'actuelle «bureaucratisation » comme un pendant de la culture néolibérale et de sa diffusion dans l'administration publique. Pour ces auteurs, le développement de la logique de marché (public) à l'œuvre focalise davantage l'attention sur le respect des procédures que sur les résultats (théoriquement) attendus.

\section{Santé au travail et efficacité des associations, les conséquences de la bureaucratisation}

L'enquête révèle que l'environnement des centres sociaux, marqué par le morcellement, l'incertitude et la tendance à la bureaucratisation, a des conséquences sur le travail des directeurs.trices des centres sociaux. Elle fait apparaître qu'ils sont exposés aux six familles de facteurs de risques psychosociaux identifiés par Michel Gollac et Marceline Bodier (2011) comme ayant des effets sur la santé au 
travail ${ }^{7}$. Cependant, pour ne pas développer un propos trop généraliste, nous proposons ici de présenter les trois principaux facteurs de risques auxquels sont confrontés les directeurs-trices, à savoir la perte de sens du travail, la surcharge de travail et l'exposition récurrente à des situations stressantes.
(7) Les six familles de risques psychosociauxsont:l'intensitédu travailetle tempsdetravail;lesexigencesémotionnelles;l'autonomie;les rapportssociaux autravail;lesconflitsdevaleurs;linsécuritéde la situation de travail.

Facteurs de risques psychosociaux dont les aspects positifs (protection) et négatifs (exposition) ont été relevés dans l'enquête réalisée auprès des directeurs-trices de centres sociaux

\begin{tabular}{|c|c|c|}
\hline $\begin{array}{l}\text { Facteurs de risques psychosociaux (ou de bien-être } \\
\text { au travail sous certaines conditions) }\end{array}$ & $\begin{array}{c}+ \\
\text { (facteurs de protection) }\end{array}$ & $\begin{array}{c}- \\
\text { (facteurs d'exposition) }\end{array}$ \\
\hline \multicolumn{3}{|l|}{ Intensité du travail et temps de travail } \\
\hline Intensité et complexité du travail & & $\mathbf{x}$ \\
\hline Contraintes de rythme & & $\mathbf{x}$ \\
\hline Objectifs irréalistes ou flous & & $\mathbf{x}$ \\
\hline Polyvalence & $\mathbf{x}$ & $\mathbf{x}$ \\
\hline $\begin{array}{l}\text { Exercice de responsabilités (en cas de manque de } \\
\text { moyens pour les exercer de façon convenable) }\end{array}$ & $\mathbf{x}$ & $\mathbf{x}$ \\
\hline Injonctions contradictoires & & $\mathbf{x}$ \\
\hline Interruptions d’activité & & $\mathbf{x}$ \\
\hline Sous-qualification (par rapport à ce qui est demandé) & & $\mathbf{x}$ \\
\hline $\begin{array}{l}\text { Temps de travail (durée, extension de la disponibilité, } \\
\text { conciliation travail-hors travail) }\end{array}$ & & $\mathbf{x}$ \\
\hline \multicolumn{3}{|l|}{ Exigences émotionnelles } \\
\hline Relation au public & $\mathbf{x}$ & $\mathbf{x}$ \\
\hline Contact avec la souffrance & & $\mathbf{x}$ \\
\hline Peur (de violences externes, d’un échec...) & & $\mathbf{x}$ \\
\hline \multicolumn{3}{|l|}{ Autonomie } \\
\hline Autonomie procédurale, temporelle & $\mathbf{x}$ & $\mathbf{x}$ \\
\hline Prévisibilité du travail, possibilité d’anticiper & & $\mathbf{x}$ \\
\hline Développement des compétences & $\mathbf{x}$ & \\
\hline Monotonie ou plaisir au travail & $\mathbf{X}$ & \\
\hline \multicolumn{3}{|l|}{ Rapports sociaux au travail } \\
\hline Justice, reconnaissance & $\mathbf{x}$ & $\mathbf{x}$ \\
\hline Soutien des collègues & $\mathbf{x}$ & $\mathbf{x}$ \\
\hline Equilibre effort-récompense & $\mathbf{x}$ & $\mathbf{x}$ \\
\hline Coopération & $\mathbf{x}$ & $\mathbf{x}$ \\
\hline Intégration dans un collectif & $\mathbf{x}$ & $\mathbf{x}$ \\
\hline Soutien technique de l'encadrement & & $\mathbf{x}$ \\
\hline Reconnaissance par le public & $\mathbf{x}$ & \\
\hline Valorisation sociale du métier & & $\mathbf{x}$ \\
\hline Violences internes, tensions & & $\mathbf{x}$ \\
\hline \multicolumn{3}{|l|}{ Conflits de valeurs } \\
\hline Conflits éthiques & $\mathbf{x}$ & $\mathbf{x}$ \\
\hline Qualité empêchée & $\mathbf{x}$ & $\mathbf{x}$ \\
\hline Travail inutile & & $\mathbf{x}$ \\
\hline \multicolumn{3}{|l|}{ Insécurité de la situation de travail } \\
\hline Sécurité (de l’emploi, du salaire, de la carrière) & & $\mathbf{x}$ \\
\hline $\begin{array}{l}\text { Soutenabilité du travail (possibilité de le faire tout au } \\
\text { long de sa carrière professionnelle) }\end{array}$ & $\mathbf{x}$ & $\mathbf{x}$ \\
\hline
\end{tabular}




\section{Perte de sens : «Empiétement du rôle de gestionnaire sur celui de chef de projet"}

Il est tout d'abord remarquable que ce phénomène a des conséquences sur le « sens du travail », qui est l'un des principaux sujets de satisfaction évoqués par les directeurs·trices de centres sociaux. Ils déplorent le fait que l'environnement public et ses évolutions pèsent sur leur métier. "En tant que directrice, je ne trouve plus de temps pour construire un projet sereinement, jele passe en paperasserie administrative, qu'elle soit liée aux financements, à la comptabilité, à la GRH ou aux normes et procédures en tout genre. " "Le temps de travail consacréaux tâches administratives, dossiers, bilans, enquêtes, consultations de toutes sortes est sans cesse à la hausse, ce qui fait que je passe désormais deux bons tiers de mon temps sur mon PC à faire de la gestion et non plus de l'animation d'équipe ni du pilotage de projet.» "On assiste à une dérive de la fonction. Le directeurn'est plus un animateur de projet, mais plutôt un gestionnaire de dispositifs. »

Les directeurs-trices déplorent leur éloignement de ce qui fait, de leur point de vue, le sens et l'objet de leur métier. Ils regrettent "l'empiétement du rôle de gestionnaire sur celui de chef de projet ».

\section{Charge de travail importante}

Si les évolutions de l'environnement institutionnel des centres sociaux ont des conséquences sur le sens du travail, elles ont aussi un impact sur le temps de travail, en termes de durée et d'empiétement sur la vie privée. "La pression sur les moyens augmentant, l'ensemble de nos ressources sont mobilisées pour les habitants. Je n'ai plus de secrétariat, et cela manque beaucoup. Je travaille en moyenne 60 heures par semaine, sans compter les réunions le soir (élus, administrateurs, conférences, voux, etc.) ou les week-ends travaillés (activités, événements et manifestations, inaugurations, etc.)."

"Charge de travail extrêmement importante, difficulté de concilier l'implication professionnelle avec des horaires souvent décalés et la vie defamille. " "Obligation de devoir travailler sur de l'administratifen dehors du cadre du travail (soirées, congés, week-end). " Impossible de prendre mes congés en 2017. » «La journée n’est jamais finie. » «Un rythme important et dense avec un travail en soirée important." "Enfin, sur un plan personnel, l'insatisfaction naît de mes difficultés à concilier vie professionnelle et vie personnelle : trop de temps au travail, pas assez de temps pour soi. »

Ainsi, les mutations institutionnelles et la bureaucratisation à l'œuvre ont des conséquences sur la charge de travail des directeurs.trices. Ce qui n'est évidemment pas neutre pour leur bien-être au travail.

\section{Les situations de pression et de stress deviennent la norme}

La bureaucratisation alourdit la charge de travail des directeurs·trices, entraînant des effets sur leur santé. Ils évoquent des situations de pression, de tension, de stress permanent. "Augmen- 
tation de la charge mentale : demande d'adaptation perpétuelle et contraintes organisationnelles permanentes, pression de la rentabilité, augmentation des critères d'exigence, charge de travail et durée du travail en hausse constante, exigences émotionnelles (amabilité vis-à-vis des usagers, du CA, des partenaires, disponibilitéconstante, sentiment de devoir "obéir"...). » "La démultiplication des dossiers de subventions, appels à projets, mécénat engendre une pression permanente et une intensité cérébrale qui peut amener à une saturation (burn-out...). " "Tout va beaucoup trop vite : je trouve que le rythme n'est pas tolérable. " «Pression constante (partenaires, élus, échéances, gestion). " "Le niveau de stress est toujours en augmentation. " "Le sentiment de ne jamais terminer la "to do list", qui ne cesse de s'allonger. » Comme le montrent ces extraits d'entretiens, la bureaucratisation des relations entre associations et tutelles a des conséquences sur la charge de travail des directeurs.trices d'associations. Cette charge, à laquelle s'additionnent des modalités et des temporalités différentes, est facteur de stress pour ces dirigeant·es.

\section{Une situation qui n'est pas isolée}

Cette enquête portait spécifiquement sur la branche Alisfa - dans laquelle, d'ailleurs, les directeurs.trices d'établissements d'accueil de jeunes enfants sont confrontés aux mêmes problématiques que les directeurs-trices de centres sociaux, bien que les financeurs ne soient pas les mêmes - mais Chorum a également réalisé d'autres enquêtes similaires, l'une en direction de directeurs-trices d'associations d'aide à domicile, en octobre 2013, et une autre en direction des directeurs-trices d'ateliers et de chantiers d'insertion, en mars 2018.

Ces associations présentent des caractéristiques différentes de celles de la branche Alisfa, mais sont, elles aussi, fortement soumises aux politiques publiques en matière de financement. Les directeurs y sont également " multi-casquettes », pilotes de l'activité sans ressources techniques en interne.

Dans ces deux études, on observe des situations qui présentent des similitudes avec celles des directeurs-trices de la branche Alisfa : exposition aux RPS liée notamment aux financements, aux contraintes budgétaires et économiques, aux injonctions institutionnelles, etc.

\section{Vers un renforcement des contraintes?}

Nous avons vu que les directeurs-trices de centres sociaux sont en première ligne face à la complexité de leur environnement institutionnel. Ils subissent de plein fouet les mutations de l'action publique (Cottin-Marx et al., 2017), sa marchandisation et sa bureaucratisation, qui s'ajoutent à son morcellement.

Ainsi, il semble que les engagements répétés de l’État et des collectivités locales à «donner cohérence et visibilitéà la politique associative $^{8}$ ", à « simplifier» ou à " moderniser ${ }^{9}$ » l'action publique soient restés lettre morte.

Si le présent article rend compte des effets de l'environnement public des associations sur les dirigeants de ces structures, il ne
(8) Charte des engagements réciproques entre l'État, le Mouvement associatif et les collectivités territoriales, février 2011.

(9) Le 28 mars 2013, le président de la République, François Hollande, annonçait aux Français un «choc de simplification ». Un chantier qu'il engageait dèsle 14 mai, lors d'unepremière réunion sur la simplification des normes et des procédures administratives, mobilisant les membres du gouvernement. Le 17 juillet 2013 , le gouvernement dévoilait les 200 mesures de son programme de sim- 
faut pas sous-estimer les conséquences affectant l'ensemble des travailleurs, qu'ils soient salariés ou bénévoles (Simonet, 2010). Nous pouvons notamment émettre l'hypothèse que cela explique en partie les difficultés rencontrées par les associations employeuses quand elles doivent renouveler leurs instances dirigeantes bénévoles.

Si les pouvoirs publics ne sont pas, au sens strict, "les patrons des associations " (Mouvements, 2015), leur position de donneurs d'ordre (à des associations reléguées au rang de prestataires) et les contraintes qu'ils font peser sur les associations semblent se renforcer. $\mathrm{Si}$, aux yeux de la loi, ils ne sont pas encore responsables des risques psychosociaux qu'ils font naître, il paraît souhaitable qu'une réflexion se lance (et aboutisse à des effets rapides et concrets) en s'inspirant de la philosophie de la loi du 27 mars 2017 relative au "devoir de vigilance des sociétés mères et des entreprises donneuses d'ordre », qui fait obligation à ces dernières de prévenir les risques sociaux, environnementaux et de gouvernance liés à leurs opérations. 


\section{BIBLIOGRAPHIE}

Bezes P. et Siné A., 2011, " Gouverner (par) les finances publiques. Perspectives de recherche", dans Bezes P. (dir.), Gouverner (par) les finances publiques, Paris, Presses de Sciences Po, p. 17-111. Cortesero R., 2013, « Les centres sociaux, entre participation et cohésion sociale ", dossier d'études n 160 , Cnaf.

Cottin-Marx S., 2016, Professionnaliser pour "marchandiser» (et inversement), thèse de l'université Paris Est.

Cottin-Marx S., Hély M., Jeannot G. et Simonet M., 2017, « La recomposition des relations entre l'État et les associations : désengagements et réengagements ", Revue française d'administration publique, $\mathrm{n}^{\circ} 163$, p. 463-476.

Cottin-Marx S., Sociologie du monde associatif, Paris, La Découverte, coll. « Repères », 2019.

Durand R., 2006, Histoire des centres sociaux, Paris, La Découverte.

Graeber D., 2015, Bureaucratie. L'utopie des règles, Paris, Les Liens qui libèrent.

Gollac M. et Bodier M., 2011, " Mesurer les facteurs psychosociaux de risque au travail pour les maîtriser ", rapport du collège d'expertise sur le suivi des risques psychosociaux au travail, pour le ministère du Travail, de l'Emploi et de la Santé.

Hall M., Barr C., Easwaramoorthy M., Wojciech Sokolowski S. et Salamon L., 2005,
Analyse comparative du secteur sans but lucratif et bénévole au Canada, Toronto, Imagine.

Hély M., 2009, Les Métamorphoses du monde associatif, Paris, Presses universitaires de France. Hibou B., 2012, La Bureaucratisation du monde à l'ère néolibérale, Paris, La Découverte.

Hibou B., 2013, La Bureaucratisation néolibérale, Paris, La Découverte.

Lebon F., 2016, " De la démocratie en centre social. Enquête sur les relations professionnelles dans une association de quartier ", Recma, $\mathrm{n}^{\circ} 340$, p. 93-108.

Lipsky M., Smith S. R., 1989-1990, « Nonprofit organizations, government, and the welfare state ", Policital Science Quarterly, vol. 104, n 4. Mouvements , $\mathrm{n}^{\circ}$ 81, 2015, "Qui est le patron des associations?».

Simonet M., 2010, Le Travail bénévole. Engagement citoyen ou travail gratuit?, La Dispute.

Tchernonog V. et Prouteau L., 2017, « Évolutions et transformations des financements publics des associations ", Revue française d'administration publique, ${ }^{\circ} 163$, p. 531-542.

Tchernonog V. et Prouteau L., 2019, Le Paysage associatiffrançais, Paris, Dalloz, Juris Édition.

Thoenig J.-C., 2005, «Territorial administration and political control : Decentralization in France ", Public Administration, vol. 83, p. 685-708. 